\title{
Quantitative Oxidation State Analysis of Transition Metals in a Lithium-ion Battery with High Energy Resolution AES
}

\author{
A. Tanaka, K. Tsutsumi, H. Onodera and T. Tazawa \\ JEOL 1td., Musashino 3-1-2, Akishima, Tokyo, Japan
}

After a first suggestion of the use of lithium transition-metal oxides in the cathode of lithium-ion battery (LIB) [1], many researchers have investigated to improve its performance such as higher-energy density, longer-life, and lower-cost. For the systematic, effective development, various transition-metals for the cathode active material have been investigated and chemical state characterization have earnestly been desired of small particles with a size of less than one micrometer.

Auger electron spectroscopy (AES), which is well-known as a powerful method to detect lithium like Xray photoelectron spectroscopy (XPS), is best suited for the above purpose. Auger spectra have been known to have chemical state information as a peak shift and a peak shape change [2], and the chemical state analysis based on standard spectra measured with high energy resolution of $\Delta \mathrm{E} / \mathrm{E}=0.1 \%$ is an effective method for complex shape Auger spectra [3].

In this study, we focused on the manganese oxides which have been one of the attracted materials as a low-cost cathode active material for a LIB. The previous attempt to identify oxidation state of manganese with AES did not lead to a fruitful result [4]. The reason is that chemical state analysis was mainly based on the shift of the peak positions of the Mn MVV spectra. Unfortunately no appreciable change in the peak positions was observed due to oxidation states of manganese. Beside, the Mn MVV spectrum is situated in the low energy region between 30 and $100 \mathrm{eV}$ where the spectra of many other elements appear, which fact may cause difficulty in the separation of manganese components from superimposed spectra. In the application to the LIB, especially, the Mn MVV spectrum inevitably overlaps with the lithium spectrum because the peak positions of Mn MVV and Li KVV are located at around $50 \mathrm{eV}$. In this presentation, the Mn LMM spectra of three oxidation states: $\mathrm{Mn}^{0}(\mathrm{metal})$, $\mathrm{Mn}^{2+}(\mathrm{MnO})$, and $\mathrm{Mn}^{4+}\left(\mathrm{MnO}_{2}\right)$ were measured for standard spectra, using high purity metal and oxides, with a higher energy resolution of $0.1 \%$ with a commercial Auger electron spectrometer equipped with a field emission gun and a hemi-spherical analyzer with variable energy resolution (0.05-0.5\%): JAMP9510F (JEOL Ltd.). These were added to our file of standard spectra, consisting of a large number of spectra of elements with well defined chemical states. No noticeable differences were detected in their peak positions, but appreciable differences were observed in their shapes. The latter differences were large enough for superimposed spectra to be separated into each individual oxidation state spectrum as shown in Fig.1.

Moreover, we applied the chemical state analysis with the standard spectra to the depth profile of composition result for two different types of particle used as a cathode active material: one contains an appreciable amount of lithium, the other contains less lithium, with an energy resolution of $0.1 \%$. Fig. 2 shows that the oxidation state of manganese in the particle containing lithium was mainly $\mathrm{Mn}^{4+}$, whereas that of manganese in the particle containing less lithium was mainly $\mathrm{Mn}^{2+}$. The oxidation state difference due to the amount of lithium was observed for each particle. 
The present study confirms the very high potential of our recently developed method of oxidation state analysis with higher energy resolution AES. Moreover we will discuss the possibility of quantitative analysis of chemical state of manganese by the use of the absolute intensity of standard spectrum[3].

\section{References:}

[1] J.B. Goodenough et al., US patent, 4302518(1981)

[2] Madden, H. H., Journal of Vacuum Science and Technology, Vol.18 (1981), p.677

[3] K.Tsutsumi et al., Journal of the Surface Science Society of Japan Vol.33 (2012), p.431

[4]S. Singh et al., Appl. Surf. Sci. 152 (1999), p. 213

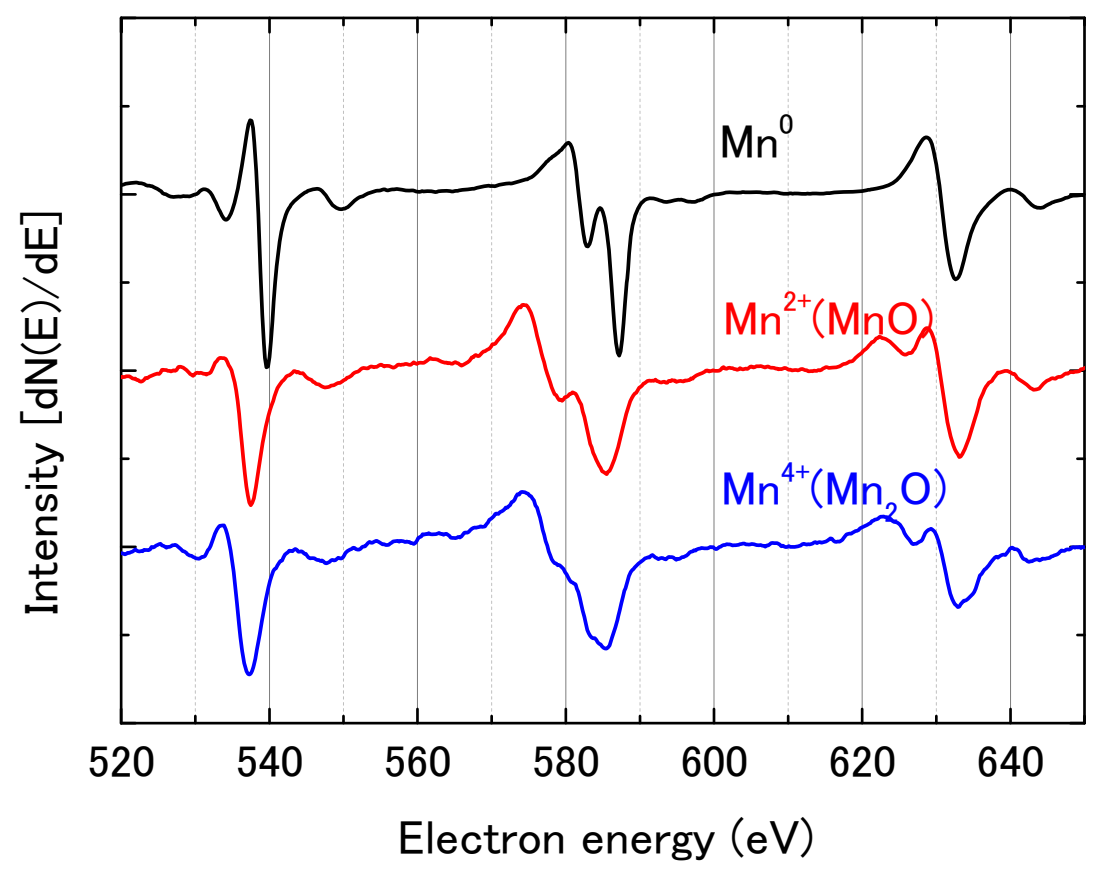

Figure 1. Mn LMM spectra of $\mathrm{Mn}^{0}$ (metal), $\mathrm{Mn}^{2+}(\mathrm{MnO})$ and $\mathrm{Mn}^{4+}\left(\mathrm{Mn}_{2} \mathrm{O}\right)$
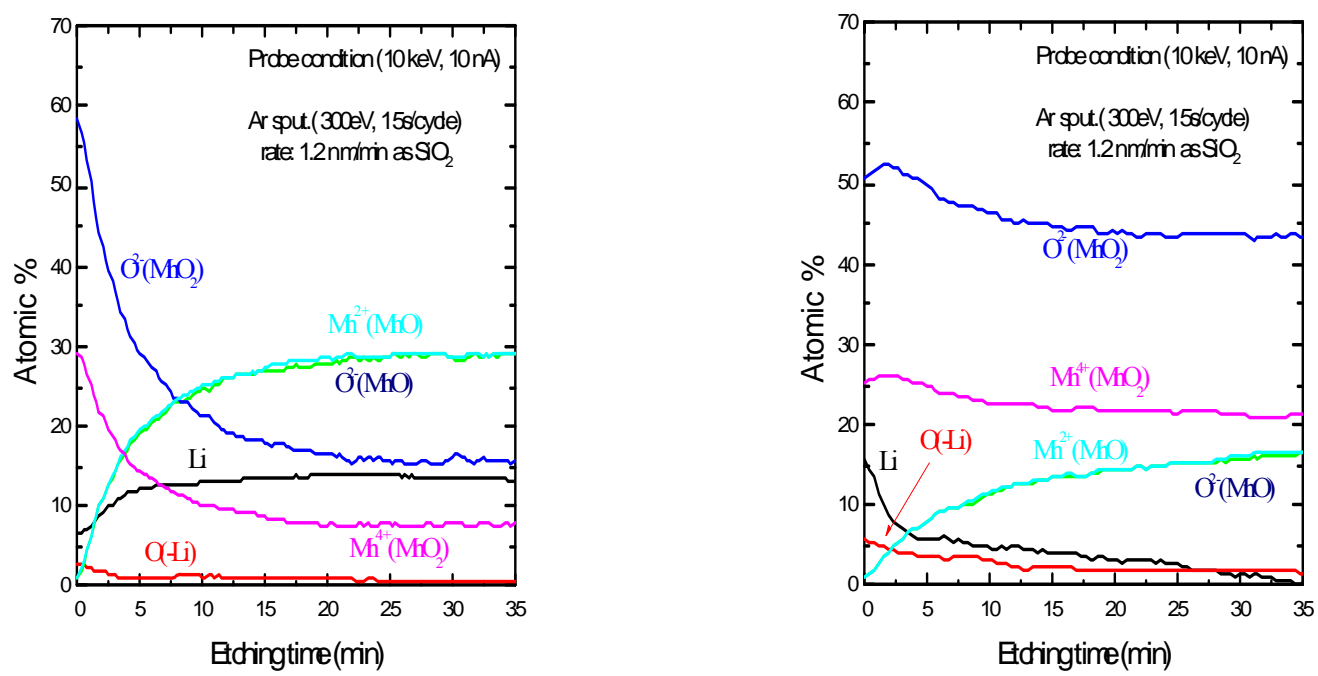

Figure 2. The depth profile of the atomic concentration of each chemical state for two different types of particle used as a cathode active material 